\title{
Slow dynamics and strong finite-size effects in many-body localization with random and quasiperiodic potentials
}

\author{
Felix Weiner, ${ }^{1}$ Ferdinand Evers, ${ }^{1}$ and Soumya Bera ${ }^{2}$ \\ ${ }^{1}$ Institute of Theoretical Physics, University of Regensburg, D-93040, Germany \\ ${ }^{2}$ Department of Physics, Indian Institute of Technology Bombay, Mumbai 400076, India
}

(Received 25 May 2019; revised manuscript received 22 August 2019; published 24 September 2019)

\begin{abstract}
We investigate charge relaxation in disordered and quasiperiodic quantum wires of spinless fermions $(t-V$ model) at different inhomogeneity strength $W$ in the localized and nearly localized regime. Our observable is the time-dependent density correlation function, $\Phi(x, t)$, at infinite temperature. We find that disordered and quasiperiodic models behave qualitatively similar: Although even at longest observation times the width $\Delta x(t)$ of $\Phi(x, t)$ does not exceed significantly the noninteracting localization length, $\xi_{0}$, strong finite-size effects are encountered. Our findings appear difficult to reconcile with the rare-region physics (Griffiths effects) that often is invoked as an explanation for the slow dynamics observed by us and earlier computational studies. Motivated by our numerical data we discuss a scenario in which the MBL-phase splits into two subphases: in $\mathrm{MBL}_{\mathrm{A}} \Delta x(t)$ diverges slower than any power, while it converges towards a finite value in $\mathrm{MBL}_{\mathrm{B}}$. Within the scenario the transition between $\mathrm{MBL}_{\mathrm{A}}$ and the ergodic phase is characterized by a length scale, $\xi$, that exhibits an essential singularity $\ln \xi \sim 1 /\left|W-W_{\mathrm{c}_{1}}\right|$. Relations to earlier numerics and proposals of two-phase scenarios will be discussed.
\end{abstract}

DOI: 10.1103/PhysRevB.100.104204

\section{INTRODUCTION}

The interplay of quantum interference and interactions exhibits some of the most fascinating phenomena to be encountered in many-body systems. A typical representative of concern to us here is many-body localization [1,2] (MBL), which has attracted considerable attention over the last decade [3-13]. At its heart is the basic observation that Anderson localization prevalent in low-dimensional, disordered systems of noninteracting fermions can be robust against interaction effects at finite temperature despite dephasing as is illustrated in Fig. 1.

Starting from the MBL phase, with decreasing disorder a quantum-critical point is reached separating the MBL region from an ergodic one. Much progress has been made in recent years with respect to experimental investigations of the corresponding localization-delocalization transition in diverse setups including cold atoms [14-19], nuclear spin chains [20], Yb ion system [21], dipolar spins [22], or in InO thin films [23]. Importantly, in several experiments a very pronounced slowdown of the relaxation dynamics has indeed been reported, when the (quasi)disorder strength exceeds a threshold value $[16,17,19]$.

The theoretical investigation of MBL and the related effects poses great challenges. On the one hand the phenomenon escapes controlled analytical investigations. On the other hand, the computationally available window of observation times, $t_{\mathrm{obs}}$, and system sizes often is too small in order to predict with confidence the thermodynamic, long-time asymptotics. In fact as shown recently, due to dynamical slowing down, even in computational studies of the ergodic regime the width of the diffusion propagator, $\Delta x(t)$, is hardly ever seen to exceed the noninteracting localization length, $\xi_{0}$, significantly
[24]. The resulting difficulties to safely extrapolate plague virtually all computational studies of dynamical behavior near and in the MBL phase. In fact, even present-day experimental studies do not (yet) allow to go very deeply into the asymptotic regime.

It is noteworthy that in computations in addition to achieving sufficiently large system sizes and long observation times

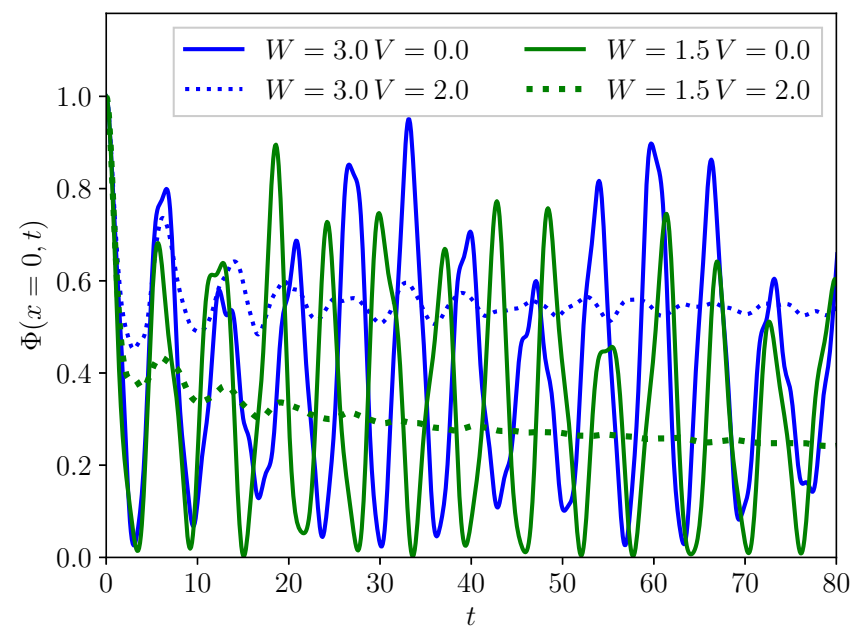

FIG. 1. Return probability inside a disordered quantum wire $(t-V$ model) with and without two-body interactions (dashed vs solid traces) illustrating the phenomenon of MBL. Two disorder strengths are shown situated in the thermalizing phase (green, $W=1.5$ ) and closer to a possibly localized phase (blue, $W=3.0$ ). While interactions wash out the mesoscopic fluctuations very rapidly (strong dephasing), the time-averaged return probability, i.e., localization, is affected only weakly (green) or hardly at all (blue). 
other difficulties arise; we list two prominent examples: (i) Computer simulations carry nonphysical numerical parameters that need to be converged, such as the bond dimension, time increments, and number of Chebyshev polynomials. Convergence can be difficult to achieve and will, in general, be sensitive to the calculated observable and the physical parameter regime. (ii) Quenches may produce transients or even asymptotic time traces that reflect properties of the initializing wave function more than the thermodynamic equilibrium. In this case the results of studies using different initialization, e.g., random versus staggered states, may be difficult to compare. ${ }^{1}$

Summarizing, a possibility to get stuck with transient or untypical behavior has to be accounted for. Notwithstanding these difficulties, from combining computational studies employing a variety of numerical techniques and analytical arguments based, e.g., on phenomenological renormalizationgroup treatments, a certain picture has emerged. It has been surveyed in several recent reviews [12,13,25-29]. In this work we present a computational study of the MBL region employing the $t-V$ model with fully random and quasiperiodic (Andre-Aubry-type) potentials [30-34]. We invoke as observable the density-density correlation function, $\Phi(x, t)$. As a first characterization its variance, $\Delta x(t)$, and the associated exponent function

$$
\beta(t)=\frac{d \ln \Delta x(t)}{d \ln t} .
$$

is being analyzed. Even though the diffusion propagator $\Phi(x, t)$ has been studied before, $[28,29,35,36]$ it is not the usual object of investigation. When the exponent $\beta$ has been considered, it has mostly been derived from closely related observables, such as the frequency-dependent conductivity at zero wave number calculated in finite-size systems [37-41]. The density response at wave number $q=\pi$ (imbalance) and the corresponding dynamical exponent $\tilde{\beta}$ has been observed more frequently $[28,42]$, though its relation to $\beta$ is not well understood, in general. Also the exponent of the return probability, $\alpha$, has been employed, although the relation to $\beta$ involves an extra exponent, which is not known with good accuracy [24].

The interest of previous authors has been in the longtime limit, $\beta_{\infty}(W) \equiv \beta(t \rightarrow \infty)$, or closely related exponents, where $\beta$ can serve as an effective order parameter; in the ergodic region prevailing at disorder $W<W_{\mathrm{c}}$ one has $\beta_{\infty}>0$, while the MBL phase, $W>W_{\mathrm{c}}$, has been defined by $\beta_{\infty}=0$. However, the data we here report leaves open a possibility for an intermediate window, $W_{\mathrm{c}_{1}}<W<W_{\mathrm{c}_{2}}$, which constitutes within the MBL phase a subphase $\mathrm{MBL}_{\mathrm{A}}$; it exhibits a width $\Delta x(t)$ diverging in time but with a growth weaker than any power, see Fig. 2 for a sketch and Fig. 3 for illustrating data. At very large disorder, $W>W_{\mathrm{c}_{2}}$, one

\footnotetext{
${ }^{1}$ For example, Luitz et al. [66] report values for the imbalance exponent $\zeta$ that are smaller than the values detected by Doggen et al. [42] by a factor of two or more, e.g., near $W=2$. Both works have tested convergence with respect to numerical parameters, while they differ in the choice of the initial state: random [66] versus Néel [42].
}

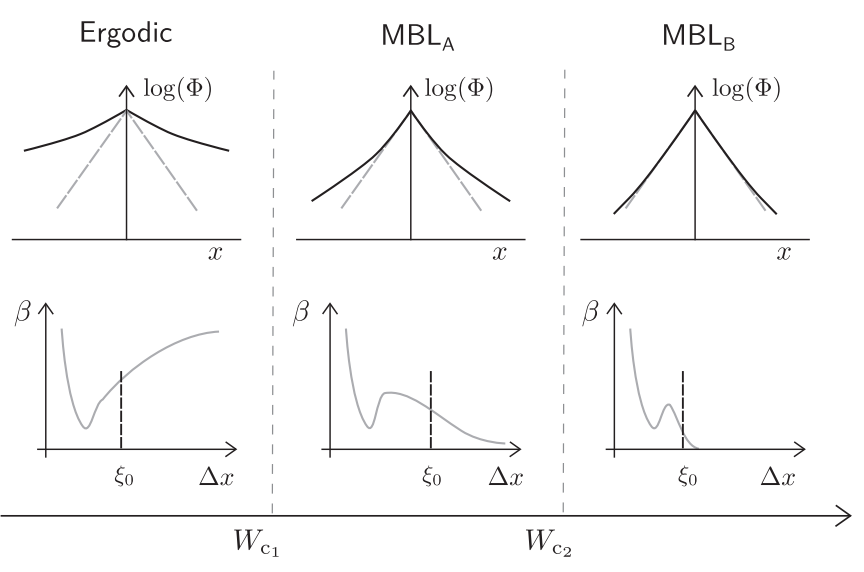

FIG. 2. Proposed infinite temperature phase diagram of the $t-V$ model indicating the possible splitting of the MBL phase into subphases. Top panel: schematic behavior of density-density correlation function $\Phi(x, t)$. Bottom panel: corresponding effective exponent $\beta$ [defined in Eq. (1)] plotted over the variance of $\Phi(x, t)$, which is $\Delta x(t) ; \xi_{0}$ indicates the non-interacting localization length. Between ergodic phase and $\mathrm{MBL}_{\mathrm{A}}, \dot{\beta}\left(x \approx \xi_{0}\right)$ changes sign; once $\mathrm{MBL}_{\mathrm{B}}$ is reached, $\beta$ vanishes at a finite $\Delta x$.

enters the subphase, $\mathrm{MBL}_{\mathrm{B}}$, in which $\beta(t) \rightarrow 0$ upon $\Delta x(t)$ approaching the localization length $\xi(W)$.

In view of the difficulty accessing the asymptotic regime of $\beta(t)$, we consider as a pragmatic indicator in addition to $\beta$ also
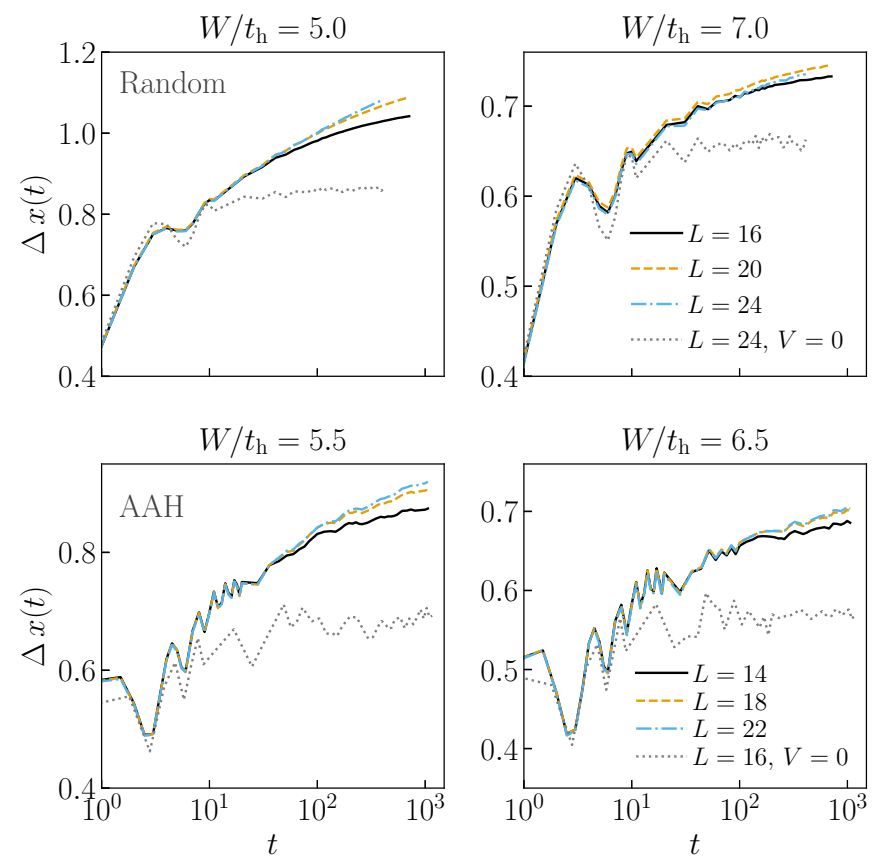

FIG. 3. Time evolution of $\Delta x(t)$ with disordered (random, top row) and quasiperiodic (bottom row) potential in $\mathrm{MBL}_{\mathrm{A}}(\mathrm{left})$ and closer to $\mathrm{MBL}_{\mathrm{B}}$ (right) phases as obtained in $t-V$ model. In $\mathrm{MBL}_{\mathrm{A}}$ a very slow growth (creep) of $\Delta x(t)$ is observed in both potential types; note that with increasing system size the slope at longest times keeps increasing, so that there is no evidence that the growth could be bounded. [Simulation parameters: $V / t_{\mathrm{h}}=2.0 ; W / t_{\mathrm{h}}=\{5.0,7.0\}$ (random); and $W / t_{\mathrm{h}}=\{5.5,6.5\}$ (quasiperiodic).] 
its slope, $\dot{\beta}$, taken at the computationally still accessible (i.e., relatively short) times $t_{\xi}$, where $\Delta x(t)$ has passed the largest relevant microscopic length. ${ }^{2}$ Following this idea, entering the $\mathrm{MBL}_{\mathrm{A}}$ phase from the ergodic side at $W=W_{\mathrm{c}_{1}}$ is signalized by a change of sign in $\dot{\beta}(t)$ at $t \gtrsim t_{\xi}$. While the ergodic phase is characterized by $\beta(t)>0, \tilde{\beta}(t)>0$, we have $\beta(t)>$ $0, \dot{\beta}(t)<0$ in $\mathrm{MBL}_{\mathrm{A}}$. A natural candidate for the microscopic length appearing in $t_{\xi}$ is the localization length, $\xi_{0}(W)$, of the noninteracting reference system, possibly having undergone an interaction-mediated (static) renormalization. Hence, we define $t_{\xi}$ implicitly via $\Delta x\left(t_{\xi}\right) \approx \xi_{0}$.

We list the salient aspects of our numerics and the scenario displayed schematically in Fig. 2: (i) In the ergodic phase, $\Delta x(t) \sim t^{\beta}$, where $\beta(t)$ converges to a constant with $\dot{\beta}(t)$ approaching zero from above; correspondingly, the slope $\dot{\beta}\left(t_{\xi}\right)$ seen in the numerical data should be positive when converged with respect to computational parameters. In $\mathrm{MBL}_{\mathrm{A}}$ the asymptotic growth of $\Delta x(t)$ is also unbound, but $\beta(t) \rightarrow 0$ with $\dot{\beta}(t)$ approaching zero from below; correspondingly, $\dot{\beta}\left(t_{\xi}\right)$ seen in the numerical data is negative when converged. Finally, in $\mathrm{MBL}_{\mathrm{B}} \Delta x(t)$ is bounded from above and $\beta$ extrapolates to zero at a finite $\Delta x$. We interpret the numerical data shown in Fig. 4 as reflecting the evolution with increasing disorder strength $W$ described here and in Fig. 2. The existence of an intermediate phase has been proposed before [43]; the relation to our scenario is discussed below.

(ii) We have applied our analysis method to the $t-V$ model with fully disordered and quasiperiodic potential. Importantly, comparing both cases we cannot detect an essential difference in the evolution of $\Delta x(t)$ with the inhomogeneity strength. Therefore, our results seem hard to reconcile with Griffithstype scenarios that rely on rare regions to explain the origin of slow dynamics $[25,27,28,44]$.

(iii) To formulate our scenario we adopt the spirit of modecoupling theory extrapolating into the asymptotic regime. It suggests that the correlation length $\xi$, when entering $\mathrm{MBL}_{\mathrm{A}}$ from the ergodic phase, exhibits an essential singularity rather than a power law, which is consistent with the Harris-Chayes criterion [45].

\section{MODEL AND COMPUTATIONAL METHOD}

We consider the $t-V$ model

$$
\begin{aligned}
\hat{\mathcal{H}} & =-\frac{t_{\mathrm{h}}}{2} \sum_{x=1}^{L-1} \hat{c}_{x}^{\dagger} \hat{c}_{x+1}+\text { H.c. }+\sum_{x=1}^{L} \epsilon_{x}\left(\hat{n}_{x}-\frac{1}{2}\right) \\
& +V \sum_{x=1}^{L-1}\left(\hat{n}_{x}-\frac{1}{2}\right)\left(\hat{n}_{x+1}-\frac{1}{2}\right),
\end{aligned}
$$

where hopping is over $L$ sites with amplitude $t_{\mathrm{h}}$. We work at a half filling and with hard-wall boundary conditions. When employing disordered potentials, the on-site energies, $\epsilon_{x}$, are

\footnotetext{
${ }^{2}$ We include $\dot{\beta}(t)$ as an indicator with the idea that $t_{\xi}$ is the only relevant time scale. Correspondingly, at $t \gtrsim t_{\xi}$ the sign of $\beta$ is converged, while it numerical value may not be, yet.
}

drawn from a box distribution $[-W, W] .{ }^{3}$ In subsequent calculations the interaction is taken twice larger, $V=2 t_{\mathrm{h}}$, than in previous studies [24] to emphasize interaction effects. At the disorder values shown in Fig. 3, the spectral statistics is close to Poissonian and in this sense the system is many-body localized, see Sec. IV C.

Our observable is the density-density correlator,

$$
\Phi(x, t)=\overline{\left\langle\left(\hat{n}_{x}(t)-\frac{1}{2}\right)\left(\hat{n}_{0}-\frac{1}{2}\right)\right\rangle} \Theta(t),
$$

with primary focus on the second moment, $\Delta x(t)=$ $\left[\sum_{x=-L / 2}^{L / 2} x^{2} \Phi(x, t)-\left(\sum_{x=-L / 2}^{L / 2} x \Phi(x, t)\right)^{2}\right]^{1 / 2}$. The disorder average is indicated by an overline; the thermal average is performed at infinite temperature and denoted by angular brackets, $\langle\ldots\rangle$; corresponding traces are performed stochastically. ${ }^{4}$ For the time evolution, Eq. (3), we employ a standard Chebyshev-polynomial propagation [46]; further details are given in Ref. [24].

\section{RESULTS}

\section{A. Simulations}

We systematically analyze traces of the kind shown in Fig. 3 in terms of the logarithmic derivative (1); the results are shown in Fig. 4. The top row displays the evolution of $\Phi(x, t)$ with system size and disorder at $t:=t_{\mathrm{obs}} \sim 400 t_{\mathrm{h}}^{-1}$. Fully consistent with our earlier findings, $\Phi(x, t)$ exhibits a pronounced non-Gaussian, nearly exponential shape-here seen in a wide range of $W$ values. Not surprisingly, the interaction effects on $\Phi(x, t)$ are strongest in the tails; they emerge gradually, with ever-increasing system size $L$ and observation time. Note that at larger disorder the interacting and noninteracting traces are seen to almost coincide for smaller distances at the time observed, see, e.g., Fig. 4(d), $W>5$. At these (possibly transient) times one has $\Delta x(t) \approx \Delta x^{(0)}$. Therefore, interaction-related static renormalization effects of the disorder strength are small at $W \gtrsim V$.

The bottom row of Fig. 4(e)-4(h) is the basis of the schematic plot Fig. 2. It shows the exponent function $\beta(t)$ that highlights the important effects related to finite $L$. Consistent with our previous findings [24], at weak disorder $W \lesssim 3$ and $\Delta x(t) \sim \Delta x_{0}$ there is a system size convergence with respect to the sign of the slope of $\beta(t)$; there is no notion of convergence at $\Delta x(t) \gtrsim \Delta x_{0}$ beyond the statement that $\beta(\Delta x)$ is curved to the right. At larger disorder, $W \gtrsim 5$, a qualitative change is observed: the curvature seen in the inset of Fig. 4(g) is about to turn left at $\Delta x(t)>\Delta x_{0}$. The MBL scenario advocated in Fig. 2 is based on the observation that the curvature of $\beta(\Delta x)$ changes sign with growing $W$, so what is a right curvature in Fig. 4 turns into a left curvature at later times; also we assume that there is no turnaround in $\beta(\Delta x)$ at even longer times, but rather a smooth decay towards the horizontal axis at $\Delta x \rightarrow \infty$. [A discussion of the pronounced

\footnotetext{
${ }^{3}$ When working with quasiperiodic potential (Aubry-André model) we choose, $\epsilon_{x}=W \cos (2 \pi \alpha x+\phi)$ with $\alpha=2 /(\sqrt{5}+1)$ and $\phi$ is the random phase distributed uniformly between $[0,2 \pi]$.

${ }^{4} \mathrm{Here}$, we have used that in the infinite temperature limit the density is homogeneous and equal to $1 / 2$.
} 

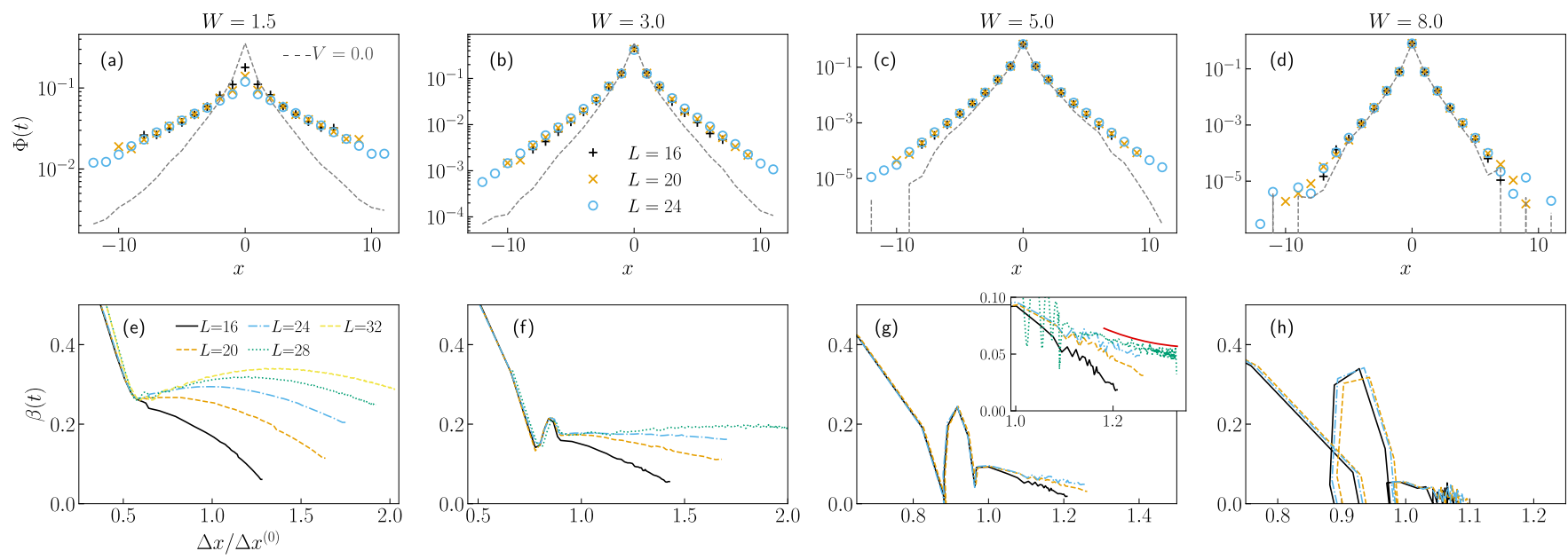

FIG. 4. Effect of interactions with increasing disorder monitored by the density-density correlation function $\Phi(x, t)$; the analogous plot for the quasiperiodic model is shown in the Appendix. Top row: $\Phi(x, t)$ for system sizes $L=16,20,24$ and disorder $W=1.5,3.0,5.0,8.0$ at $t \approx 400 t_{\mathrm{h}}^{-1}$. The noninteracting result at $L=24$ is also shown for reference (dashed line). The plot highlights how creep begins to manifest at larger disorder by lifting the tails of $\Phi(x, t)$. Bottom row: The exponent function $\beta(t)$ corresponding to first-row data plotted over $\Delta x(t) / \Delta x^{(0)}$ as suggested by Eq. (4); $\Delta x^{(0)}$ denotes the saturation width of the noninteracting diffusion propagator at long time, here taken as an indicator of $\xi_{0}$. $\beta$ inherits finite-size corrections from $\Phi(x, t)$, which persists also at larger disorder values as is seen, e.g., in the inset. The red line in there serves as guide to the eye. The evolution seen in this data condenses into the schematics displayed in Fig. 2. The noise visible at larger $W$ values reflects statistical noise from disorder averaging. The average was taken over $\sim 10000$ samples for largest system size and for largest disorder values, while for smaller system sizes $(L=16,20)$ the disorder averaging is performed over $\sim 20000$ samples.

short-time feature of the data Fig. 4(e)-4(h) bottom row, namely the spike growing in $\beta(\Delta x)$ with increasing $W$ near $\Delta x_{0}$, is given in the Appendix.]

\section{B. Phenomenological modeling}

The data for the $\beta$ function displayed in Fig. 4 condenses into the phenomenological ansatz

$$
\beta[\Delta x]=\frac{b}{1+b_{1} \mathcal{K}\left(\xi_{0} / \Delta x\right)} .
$$

Similar to our previous work [24], we here consider $\beta$ as a functional of the slowest dynamical mode, which for simplicity we parametrize with $\Delta x(t)$; the parameters $b, b_{1}$ and the kernel $\mathcal{K}$ are evolving with disorder $W$. The ansatz helps formulating a scenario for the qualitative properties of the long-time limit, which are just barely seen to emerge in Fig. 4.

Embarking on (4) we describe a passage from the ergodic regime through $\mathrm{MBL}_{\mathrm{A}}$ into $\mathrm{MBL}_{\mathrm{B}}$ with increasing $W$. The kernel $\mathcal{K}(y)$ equals unity at $y=1$ and otherwise is assumed to be a monotonous function in the interval $1 \gtrsim y \gtrsim 0$. Its precise form is evolving with the disorder strength, $W$ : With decreasing $y$, the kernel decreases in the ergodic phase, increases in $\mathrm{MBL}_{\mathrm{A}}$ and is seen to diverge at a finite $y$ upon entering $\mathrm{MBL}_{\mathrm{B}}$.

Ergodic regime $W<W_{c_{1}}$. The power-law growth of $\Delta x(t)$ characteristic for the ergodic regime is captured by (4) with $b>0, b_{1}>0$ and taking $\mathcal{K}(y)$ as decreasing to zero in the limit $y \rightarrow 0$. Namely, with the variance $\Delta x(t)$ growing in time, the kernel $\mathcal{K}\left[\xi_{0} / \Delta x(t)\right]$ vanishes and so the denominator converges to unity. At this point, $b$ should be identified with the diffusion exponent: $b=\beta_{\infty}$

Transition into $M B L_{A}$. The transition is associated with a vanishing slope, $\dot{\beta}=0$. Within in the setting (4) this requires the existence of a particular value of the disorder $W_{\mathrm{c}_{1}}$, such that the contribution of the term containing $b_{1} \mathcal{K}(\lambda / \Delta x)$ is independent of $\Delta x$. This situation could be realized in several ways; the most plausible scenario is $\left.\mathcal{K}(y)\right|_{W_{c_{1}}}=1 .{ }^{5}$ We thus parametrize $\mathcal{K}(y)$ in the vicinity of $W \approx W_{\mathrm{c}_{1}}$ as follows:

$$
\mathcal{K}(y) \approx \tilde{\mathcal{K}}(y)^{W-W_{\mathrm{c}_{1}}}, \quad \tilde{\mathcal{K}}(y):=e^{\left.\frac{d \ln \mathcal{K}(y)}{d W}\right|_{W_{\mathrm{c}_{1}}}} .
$$

This shape has consequences for the correlation lengths, $1 \approx b_{1} \mathcal{K}\left(\xi_{0} / \xi\right)$, which we here define implicitly; we have $\tilde{\mathcal{K}}\left(\xi_{0} / \xi\right) \approx b_{1}^{\frac{1}{\left(\mathrm{C}_{\mathrm{c}_{1}}-W\right)}}$. To illustrate the impact of this statement, we adopt a power-law shape $\tilde{\mathcal{K}}\left(\xi_{0} / \xi\right) \sim\left(\xi / \xi_{0}\right)^{\kappa}, \kappa>0$, so that

$$
\xi \sim \xi_{0} b_{1}^{\frac{1}{\kappa\left(W_{\mathrm{c}_{1}}-W\right)}}
$$

It is thus seen that the scenario formulated in (4) tends to generate length scales that are diverging at the transition in an exponential rather than in a power-law fashion.

$M B L_{A}$ phase. According to (5), in $\mathrm{MBL}_{\mathrm{A}}$ the kernel $\mathcal{K}(y)$ diverges with $y \rightarrow 0$ keeping $b>0$ and $b_{1}>0$. With growing time, the second term in the denominator grows without bound and eventually $\beta(\Delta x) \approx \frac{b}{b_{1}} \mathcal{K}\left(\xi_{0} / \Delta x\right)^{-1}$. The behavior is consistent with the data Fig. 4 as seen, e.g., at $W=5$. It predicts for the $\beta$ function a decay approaching zero upon $\Delta x(t) \rightarrow \infty$.

Transition into the $M B L_{B}$ phase. Upon approaching $W_{\mathrm{c}_{2}}$ and $\mathrm{MBL}_{\mathrm{B}}$ the kernel $\mathcal{K}(y)$ diverges at a nonvanishing value $y_{c}$. For illustration we adopt the generic form $\mathcal{K}(y)=\frac{y^{\alpha}}{1+b_{2} y^{1 / v}}$

\footnotetext{
${ }^{5}$ Alternative possibilities could be (i) $b_{1}\left(W_{\mathrm{c}_{1}}\right)=0$ or (ii) $b_{1}\left(W_{\mathrm{c}_{1}}\right) \rightarrow \infty$. However, in our scenario we do not follow these directions.
} 
with $\alpha, v>0$. In $\mathrm{MBL}_{\mathrm{A}}$, we have $b_{2}>0$ and so the kernel diverges in a power-law fashion, $\mathcal{K}(y) \sim y^{\alpha-1 / v}$, as required. When $W$ crosses $W_{\mathrm{c}_{2}}$ from below, the coefficient $b_{2}(W)$ changes sign from positive to negative, so in $\mathrm{MBL}_{\mathrm{B}}$ the kernel diverges at $y^{1 / \nu}=\left|b_{2}\right|^{-1}$. we conclude that the localization length diverges $\xi \approx \lambda\left|W_{\mathrm{c}_{2}}-W\right|^{-v}$.

\section{Discussion}

The model (4) has implications, which can be tested further. We mention the following two: (i) When entering $\mathrm{MBL}_{\mathrm{A}}$ from the ergodic side, $\beta_{\infty}\left(W_{\mathrm{c}_{1}}\right)$ is taking a nonvanishing, critical value. In contrast, at the critical point separating $M_{B} L_{B}$ and $\mathrm{MBL}_{\mathrm{A}} \beta_{\infty}\left(W_{\mathrm{c}_{2}}\right)=0$ is expected. (ii) Previous computational studies have attempted a scaling analysis near $W \approx W_{\mathrm{c}_{1}}$ in order to extract a localization length exponent based on a power-law dependency $\xi \sim\left|W-W_{\mathrm{c}_{1}}\right|^{-v}$. The value of $v$ has not consolidated, yet: Luitz et al. [8] obtained $v \approx 0.7$ from the system size scaling of the magnetization density, violating the Harris-Chayes bound $v>1$ in one dimension [45]. This led to a subsequent discussion [47] attempting at rationalizing this result. On the other hand, recent exact diagonalization studies of the scaling of the Schmidt gap [48] or local temperature fluctuations [49] found exponents exceeding 2, which is consistent with the Harris bound. We point out that within the scenario outlined here the transition that has been investigated in these studies would correspond to the entry into $\mathrm{MBL}_{\mathrm{A}}$, so the correlation length diverges stronger than any power. Hence, the Harris-Chayes bound is satisfied automatically.

Saying this, we emphasize once again the exploratory character of the scenario developed with Eq. (4). Due to creep, we do not see at present a reliable way of telling whether the system actually localizes or not. We stress that creep is a phenomenon that gradually emerges at largest system sizes, longest time scales, and only in the tails of the distribution (4). For observing or ruling out creep with frequently employed observables, such as the level statistics, the return probability or the sublattice imbalance, deviations have to be identified from the noninteracting background behavior; such deviations manifest as small signals towards delocalization that slowly grow with increasing time and system size and thus are challenging to consolidate.

\section{RELATION TO EARLIER STUDIES}

\section{A. RG studies}

We compare our scenario with recent renormalization group (RG) calculations [43,50-53] and a numerical study [54]. Dumitrescu et al. [43] foresee a Kosterlitz-Thoulesstype scenario that also exhibits an intermediate MBL phase; also in this case an exponentially diverging length scale was predicted at the transition into the ergodic phase (implying $v=\infty$ ), however, with an exponent $\left|W-W_{\mathrm{c}_{1}}\right|^{-1 / 2}$ (rather than $\left.\left|W-W_{\mathrm{c}_{1}}\right|^{-1}\right)$. This intermediate phase is characterized by power-law distributed rare thermal blocks with an exponent dependent on disorder strength, taking a universal value only at the critical point $[43,51,54]$; at strong disorder the distribution becomes exponential.

In a recent work [50] a modification of the $\mathrm{RG}$ scheme underlying Ref. [51] was proposed, introducing a second length scale. The intermediate phase of the original model appears to be unstable under this modification and, in fact, a power-law distribution occurs only at the critical point. Therefore, from the perspective of phenomenological RGs, the existence of an intermediate phase is still under debate. In order to clarify if creep relates to rare disorder fluctuations, we have been comparing dynamics in random and quasiperiodic potentials in Fig. 2, see also the Appendix. Remarkably, we observe a qualitatively similar slow dynamics in both cases. This points towards a possibility that rare events are not the only viable explanation for creep as observed, e.g., in Fig. 3.

Our assertion of slow dynamics receives (tentative) support from most recent experiments in optical lattices [19]. The Bose-Hubbard model was simulated with quasiperiodic disorder potential; a slow particle transport has been seen at finite system sizes $(L=8,12)$ above the critical disorder strength. We would like to interpret this observation as a possible experimental manifestation of a creep-type dynamics.

\section{B. Numerical studies}

The exponent function $\beta(t)$, Eq. (1), was considered before by Bar Lev, Cohen, and Reichman [35]. The fitted exponents together with selected raw data are shown in Fig. 3 of their work. Even though system sizes and observation times are considerably smaller than the ones used in our paper, the onset of creep at large disorder is clearly visible in their raw data, e.g., at $W \approx 7$.

One would expect that manifestations of the creep phenomenon exist in the time dependency of generic correlation functions and we believe that this indeed is the case. For instance, Doggen et al. [42] have simulated the quench of an initial state with maximum sublattice imbalance. In Fig. 2 of their work they observe a power-law decay of the imbalance with an exponent $\tilde{\beta}(W)$. Specifically, they report an exponential decrease that roughly parametrizes as $\tilde{\beta}(W) \approx$ $\tilde{\beta}(2) \exp [-(W-2)]$ in $2<W<5$; at larger $W$ the exponent has become so small that it can no longer be resolved within the error bars. We interpret this result as broadly consistent with our observations; in particular, it implies that $W_{\mathrm{c}}>5$ for the model considered and the localized phase proper, strictly speaking, is outside of the computational observation window. 6

Gopalakrishnan et al. [38] investigated the low-frequency asymptotics of the conductivity. They reported $\sigma(\omega) \sim|\omega|^{\alpha}$ with an exponent $\alpha$ varying continuously within a region in the MBL phase. At stronger disorder the agreement reflects the fact that interaction effects are almost invisible in the numerical data, so the noninteracting result $\alpha \sim 2$ is reached trivially at large $W$ within the available range of computational parameters. At weaker disorder near the border to the thermal phase, Gopalakrishnan et al. [38] give a smaller value $\alpha \sim 1$ corresponding to a logarithmic growth of $\Delta x(t)$. This is consistent with the behavior we observe in $\mathrm{MBL}_{\mathrm{A}}$ (see Fig. 2). In fact, a more recent study [40] on significantly larger system

\footnotetext{
${ }^{6}$ With respect to $W_{\mathrm{c}}$ a similar conclusion can also be drawn from the scaling of the Schmidt-gap displayed in Fig. 5 of the same work [42].
} 
sizes reports $\alpha \sim 1$ in a broad window of disorder strengths near the transition. We mention that an exponent varying continuously across the transition, $\alpha(W)$, has also been found in a self-consistent perturbation theory in disorder [55].

Similarly, also Barišić et al. [41] report values $\alpha \sim 1$ in the MBL phase, employing system sizes up to $L=28$. In their fitting, the authors are allowing for a nonvanishing residual dc conductivity, however, and indicate that finite-size corrections are not fully under control. So, we understand that the numerical value $\alpha=1$ is subject to a significant uncertainty. The same group later extended their studies to related correlation functions [29,56]. Reminiscent of creep, also in these correlators a very slow dynamics with significant finite-size effects can be identified, e.g., in Figs. 2(c), 2(d) of Mierzejewski et al. [56].

We mention that the sparsity of many-body states in Fock space can give rise to a kind of multifractality, which exhibits a nonuniversal and, in general, basis-dependent spectrum $[57,58]$. Most recent numerical work has confirmed this earlier result at least within a certain window of system sizes computationally available [59]. If and how multifractality of the many-body wave function manifests in the evolution of $\alpha(W)$ remains to be clarified.

Finally, also the study by Serbyn, Papic, and Abanin exhibits discernible finite-size effects, which may be consistent with creep [60]. Specifically, these authors study expectation values of energy-normalized overlap matrix elements of local operators. Figure 3 of this work shows that with increasing system size, $L$, the flow of the average turns around from insulating and redirects towards thermalizing behavior. The system size of turnaround, $L^{*}(W)$, is seen to grow with disorder. Since in this work only system sizes up to $L=16$ have been studied, it remains open if a finite critical value of disorder, $W_{\mathrm{c}}$, exists at which $L^{*}(W)$ diverges, and what numerical value it takes. ${ }^{7}$

\section{Further discussion: Spectral statistics and creep}

Level spacing distribution $P(s)$. We comment on the spectral statistics of the random potential and the AAH model. Figure 5 shows the evolution of the level spacing distribution $P(s)$ from weak (Wigner-Dyson statistics) to strong (Poisson distribution) disorder as it is observed in a relatively small system of size $L=16$. The data is qualitatively similar to the one presented, e.g., in Ref. [61]. It suggests that at disorder values exceeding $W \approx 3$ the $P(s)$ distribution is nearly Poisson and therefore samples are in a certain spectral sense close to the many-body localized phase. As is also seen from the Fig. 5 that statement holds irrespective of whether the potential is fully random or quasiperiodic.

Importantly, the distribution $P(s)$ undergoes an evolution with increasing system size $L$, see, e.g., Fig. 7 in Ref. [61] and Fig. 9 in Ref. [62]. At stronger disorder values and with $L$ increasing from small values, e.g., $L \sim 12$, the initial flow is towards a Poisson shape. This observation is fully consistent with-and in fact an indispensable requirement

\footnotetext{
${ }^{7}$ We express our gratitude to Ehud Altman for drawing our attention to this work.
}
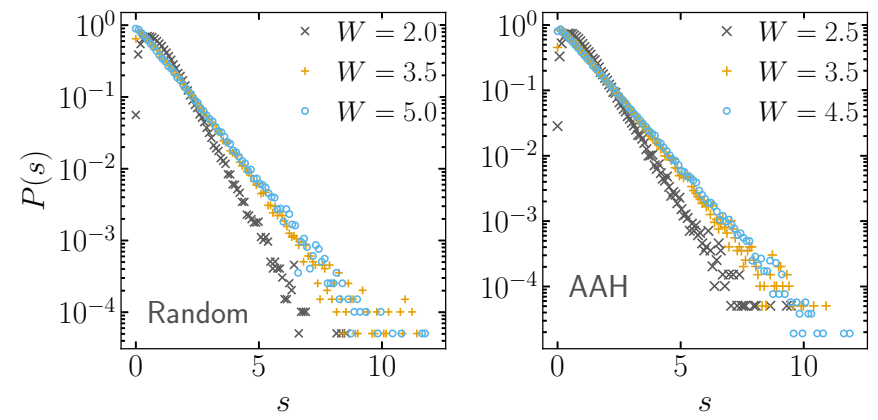

FIG. 5. Level spacing distribution for the $t-V$ model at interaction strength $V=2 t_{\mathrm{h}}$ for the fully disordered model (left, $\left.W / t_{\mathrm{h}}=2.0,3.5,5.0\right)$ and quasiperiodic (AAH) model (right, $W / t_{\mathrm{h}}=$ $2.5,3.5,4.5$ ) at system size $L=16$. Both panels show an evolution of the distribution from Wigner distribution towards Poisson statistics with increasing disorder.

for-the applicability of the concept of local integrals of motion that underlies the current understanding of many-body localization.

Notice, however, that at present there is no proof that indeed a critical value of the disorder, $W_{\mathrm{c}}$, exists, such that the asymptotic flow of $P(s)$ for the $t-V$ model or Heisenberg model is indeed towards Poisson at disorder values exceeding $W_{\mathrm{c}}$. The observation of creep indicates that the critical disorder strength, $W_{\mathrm{c}}$, may be very large; if it is finite at all, then it must correspond to a noninteracting localization length that is considerably smaller than the lattice spacing.

A conclusive numerical study of $P(s)$ to this end is lacking as of today. However, inspecting the data for $P(s)$ shown in Fig. 9 of Ref. [62] at very small $s$ and disorder, $W=4$, the flow towards Poisson indeed appears to stop already near $L \approx 18$. Conceivably, at larger $W$ a similar termination can be observed, if only at larger system sizes $L(W)$. Therefore, the present evidence concerning $P(s)$ being close to Poisson at smaller system sizes is not inconsistent with the creep scenario.

Level statistics ratio $r_{n}$. A simplified diagnostics of the level statistics is provided by the ratio $r_{n}(W, L):=$ $\min \left(\Delta_{n}, \Delta_{n+1}\right) / \max \left(\Delta_{n}, \Delta_{n+1}\right)$, where $\Delta_{n}:=E_{n}-E_{n+1}$ is the spacing between eigenvalues. For the Gaussian-orthogonal ensemble (nearly chaotic situation, $W \ll W_{\mathrm{c}}$ ) one has $r_{n} \simeq$ 0.53 while for the Poisson case (localized situation, $W \ll W_{\mathrm{c}}$ ) $r_{n} \simeq 0.39$. In analogy to conventional second-order phase transitions, it is tempting to test for single-parameter scaling near the critical point, i.e., $r_{n}(\xi / L)$, defining the localization length, $\xi \propto\left|W-W_{\mathrm{c}}\right|^{-v}$, with the corresponding exponent $v$. Indeed, the earlier attempts to determine $W_{\mathrm{c}}$ and $v$ did employ such an analysis [8].

It has been pointed out by Khemani et al. [47] already that due to the small system sizes available, typically not exceeding $L=20$, one is stuck in a preasymptotic regime. A clear illustration indicating how large the actual distance to criticality really is can be drawn, e.g., from Fig. 2(b) of Ref. [47]. A pair of traces of $r_{n}(W, L)$ for $L=12,14$ intersects near $W \approx 5.5$ while the pair $L=16,18$ intersects near $W \approx 7$. The true scaling limit would announce itself by all pairs intersecting at the same point $W=W_{\mathrm{c}}$. 
Attempting in this situation a scaling analysis and fully ignoring finite-size corrections yields $v \approx 1$. The result does not violate the Harris criterion, because the validity of the latter is restricted to the critical point, while the scaling analysis has been done in a preasymptotic regime. Following the same type of analysis, one expects with increasing system sizes a drift of $v$ and $W_{c}$ to ever larger values. Again, the existing data seems consistent with the creep scenario.

We mention that a drift in pairwise intersection points is very common in computational studies of Anderson transitions. For a recent example of how to include finite-size corrections in a case relevant to MBL, i.e., random-regular graphs, we refer to a recent study by Ref. [63].

\section{CONCLUSION}

In this work we have studied the density autocorrelation function of spinless fermions in the $t-V$ model at infinite temperature. We detect strong finite-size effects and a very slow dynamics - creep-with a tendency towards thermalization even at strong disorder, where the level statistics is close to Poissonian. Our observations are very similar for truly random and quasiperiodic potentials, so that rare-region effects seem discouraged as a likely origin of subdiffusion and creep. We believe that our results imply one out of two possibilities.

(i) Creep is a transient phenomenon, perhaps indicative of the critical fan [47] (similarly for quasiperiodic potential [33]). In this case, the width of a wave packet, $\Delta x(t)$, either grows in a power-law manner, $\Delta x(t) \sim t^{\beta}$, in a thermalizing phase, or exhibits a logarithmic (or weaker) growth window in time after which $\Delta x(t)$ eventually saturates at a finite value, $\xi(W)$, in the MBL phase.

If this is the case, an important conclusion of our study is that due to creep the asymptotic regime, $\Delta x(t) \approx \xi(W)$, in simulations is very difficult to reach in a controlled manner. We find that even at disorder values such that the noninteracting localization length is considerably smaller than the lattice spacing, $\Delta x(t)$ does not saturate within the simulation window of $1000 t_{\mathrm{h}}^{-1}$. Hence, a numerical study of the true asymptotics including $\xi(W)$, especially with respect to criticality, may have been out of reach of simulations of physical observables thus far, and, presumably, even in experiments [13].

(ii) An alternative possibility is that creep may indicate the existence of marginally thermalizing phases, such as the subphase $\mathrm{MBL}_{\mathrm{A}}$ discussed in this work. The characterizing feature is that $\Delta x(t)$ keeps growing in time weaker than any power, eventually crossing any finite upper bound $\xi$. In this case, the numerical observation of creep-type phenomena would be closer to the asymptotic regime, but the present understanding of the MBL phase would be incomplete. Conceivably, such phases can be described by complementing the concept of local integrals of motion [64,65], which has been developed for the case $W>W_{\mathrm{c}}$, with a weak integrability breaking mechanism.

\section{ACKNOWLEDGMENTS}

We thank E. Altman, I. Gornyi, V. Khemani, Z. Lenarcic, J. Moore, A. Mirlin, and D. Polyakov for inspiring discussion.
F.E. expresses his sincere gratitude to Ehud Altman and the team at UC Berkeley for their generous hospitality. S.B. would like to thank G. De Tomasi for earlier collaboration on a similar topic and for many insightful discussions. S.B. acknowledges support from DST, India, through Ramanujan Fellowship Grants No. SB/S2/RJN-128/2016 and No. ECR/2018/000876; F.E. was supported from the DFG under Grants No. EV30/11-1 and No. EV30/12-1. We acknowledge support by I. Kondov and computing time on the supercomputer ForHLR funded by the Ministry of Science, Research and the Arts Baden-Württemberg and by the Federal Ministry of Education and Research.

\section{APPENDIX A: TIME DEPENDENCE OF $\Phi(x, t)$}

In the localized phase $\Delta x(t)$ increases slowly (as shown in Fig. 2) with time above the noninteracting saturation within our observation time $\left(\sim 10^{3}\right.$ in unit of inverse $\left.t_{\mathrm{h}}\right)$. In the distribution function $\Phi(x, t)$ this propagation is reflected via the lifting of the tails with time as seen in Fig. 6. The tail lifting eventually produces a stretched-exponential shape of $\Phi(x, t)$.

\section{APPENDIX B: OSCILLATIONS IN $\beta$}

We analyze the spike observed in $\beta(\Delta x)$ (see Fig. 3), which becomes more pronounced at increasing disorder strength $W$. It occurs at $\Delta x \lesssim 1$ (cf. Fig. 7), and therefore we interpret it as a signature of the lattice spacing, which is is taken as the unit of length. In essence, the spike is a manifestation of strong oscillations that induce a nonmonotonous growth of $\Delta x(t)$ in time. Such oscillatory behavior arises from neighboring pairs of sites that have a common local potential, which deviates strongly from the environment and thus is forming a trap. The trap constitutes a two-site system with an associate energy doublet that is split by the hopping term. The dynamics of the associated charge density exhibits a characteristic frequency $\omega \approx t_{\mathrm{h}}=1$. The corresponding oscillations in the correlator $\Phi(x, t)$ are illustrated in Fig. 7 for a given disorder configuration with trap near $x=0$. Strong oscillations are also seen in Fig. 1 differing from Fig. 8 by rescaling the on-site potential to smaller values with factor $3 / 5$ (blue) and $1.5 / 5$ (red).
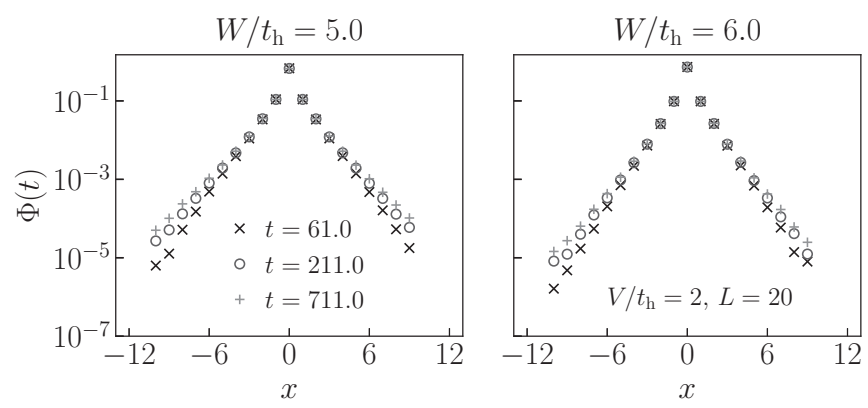

FIG. 6. Time evolution of the full distribution function $\Phi(t)$ for the random model at strong disorder. The slow dynamics manifests as tails lifting away from the nearly exponential shape that is indicative of the short-time behavior and the noninteracting limit. The data is shown for $L=20, V / t_{\mathrm{h}}=2$ and two different disorder values $W / t_{\mathrm{h}}=5.0,6.0$. 

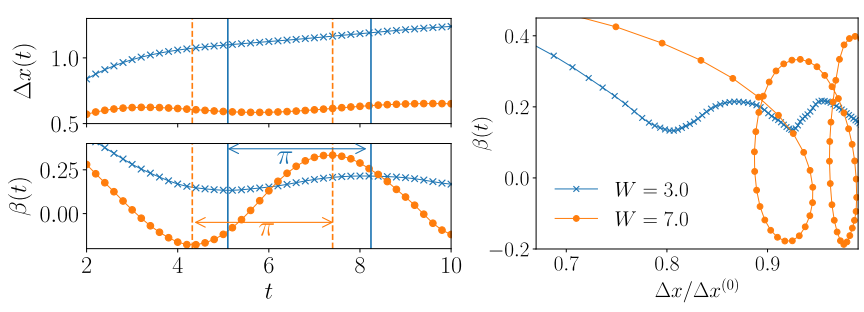

FIG. 7. Short-time behavior of $\Delta x(t)$ and $\beta(t)$ averaged over several thousand samples. Figure is illustrating the origin of the peak-structure seen in Fig. 3 of the main text at intermediate (blue) and strong (orange) disorder: $W=3.0,7.0$. Left: $\Delta x(t)$ exhibits oscillatory behavior that is emphasised in its logarithmic derivative, $\beta(t)$. At strong enough disorder, $W>W^{*}, \Delta x(t)$ becomes nonmonotonous. Vertical lines indicate a half-period of the oscillation. Right: Multivalued $\beta(\Delta x)$ at $W>W^{*}$.

We discuss the effect of interactions on time-dependent fluctuations in $\Delta x(t)$ : In the noninteracting limit, $V=0$, pronounced oscillations are seen large disorder (corresponding to strength $W=2$ ) in Fig. 8; they persist up to very long times. With decreasing disorder, Fig. 1, lower frequencies are typically mixing in because resonances between more distant sites become accessible, converting oscillations to mesoscopic fluctuations. This type of oscillation survives the ensemble average, because return times tend to be integer multiples of $2 \pi / t_{\mathrm{h}}$.

With finite interaction the random potential will, in general, undergo a static renormalization; it shifts fluctuation frequencies without affecting the qualitative physics. New effects can be expected from dynamical renormalizations, i.e., from dephasing; it is a characteristic feature of many-body systems expressing the possibility of particles to scatter off each other and thus exchanging momentum and energy. In the correlator $\Phi(x, t)$ dephasing manifests itself in a damping
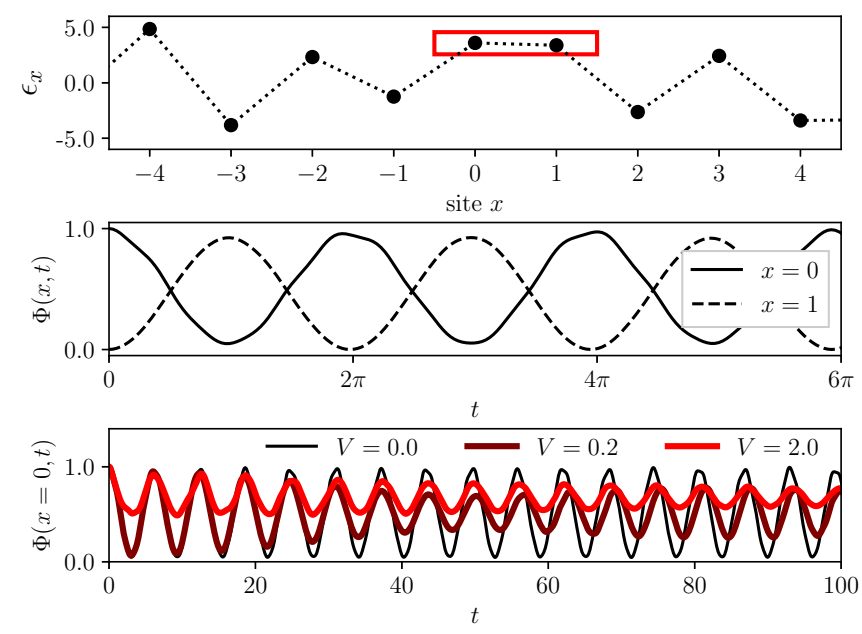

FIG. 8. Behavior of $\Phi(x, t)$ around $x=0$ for a given disorder configuration. Note that $\Phi(x, t=0)=\delta_{x, 0}$, by definition. Top: Disorder potential $\epsilon_{x}$ around $x=0$ : sites $x=0,1$ are very close in energy. Center: Coherent oscillations between neighboring sites $x=0,1$ in the noninteracting limit $V=0$. The period is close to $2 \pi$. Bottom: Damping for finite interaction strength $V$.

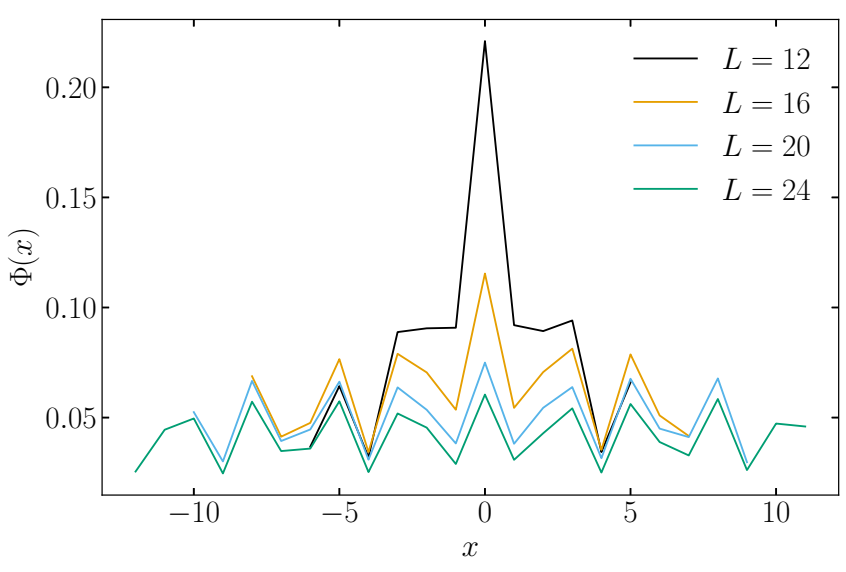

FIG. 9. $\Phi(x, t)$ at moderate strength of the quasiperiodic potential displayed at a very large observation time $t \sim 10^{3} t_{\mathrm{h}}^{-1}$ for system sizes $L=12,16,20,24$.

of the mesoscopic fluctuations, see Figs. 8, bottom, and 1 . The defining characteristics of the MBL phenomenon is that despite mesoscopic fluctuations washing out very quickly with increasing $V$, the average return probability is seen to keep values of order unity that hardly decay at all, see Figs. 8 and 1.

\section{APPENDIX C: EVOLUTION OF $\Delta x(t)$ IN A QUASIPERIODIC (AAH) POTENTIAL}

In the main text we have analyzed the evolution of the correlator $\Phi(x, t)$ for the case of fully disordered potentials, Fig. 3. We here display the analogous data obtained for the quasiperiodic case.

Thermalizing regime. Before we discuss the main data set, Fig. 10, we report an effect that we observe for quasiperiodic potentials, which is not seen in the fully random case. In Fig. 9 we show the correlation function $\Phi(x, t)$ at very large times. Despite the fact that the dynamics has explored the full system size, oscillations are seen that do not show a tendency to equilibrate, see also Fig. 10 for other disorder values. While a detailed study of this effect is beyond the scope of this work, a few comments are in place.

A preliminary analysis suggests that the amplitude of the oscillations decays with increasing system size in a power-law fashion; therefore, we tentatively consider them as a finite-size effect. Presumably, the oscillations are related to the correlations in the underlying quasiperiodic potential; hence, one would expect that their shape is sensitive to the choice of the parameter $\alpha$ in the definition of $\epsilon_{x}$ (see footnote ${ }^{8}$ for further details). Furthermore our data Fig. 10 indicates that due to these oscillations at moderate disorder $W=1.5-3.0$, i.e., in a regime that is believed to be thermal, finite-size effects can be strong. We believe that they should be observable, in principle, also in typical cold-atom experiments [16]. Finally, in recent computational work it was found that the critical

\footnotetext{
${ }^{8}$ When working with quasiperiodic potential (Aubry-André model) we choose, $\epsilon_{x}=W \cos (2 \pi \alpha x+\phi)$ with $\alpha=2 /(\sqrt{5}+1)$ and $\phi$ is the random phase distributed uniformly between $[0,2 \pi]$.
} 

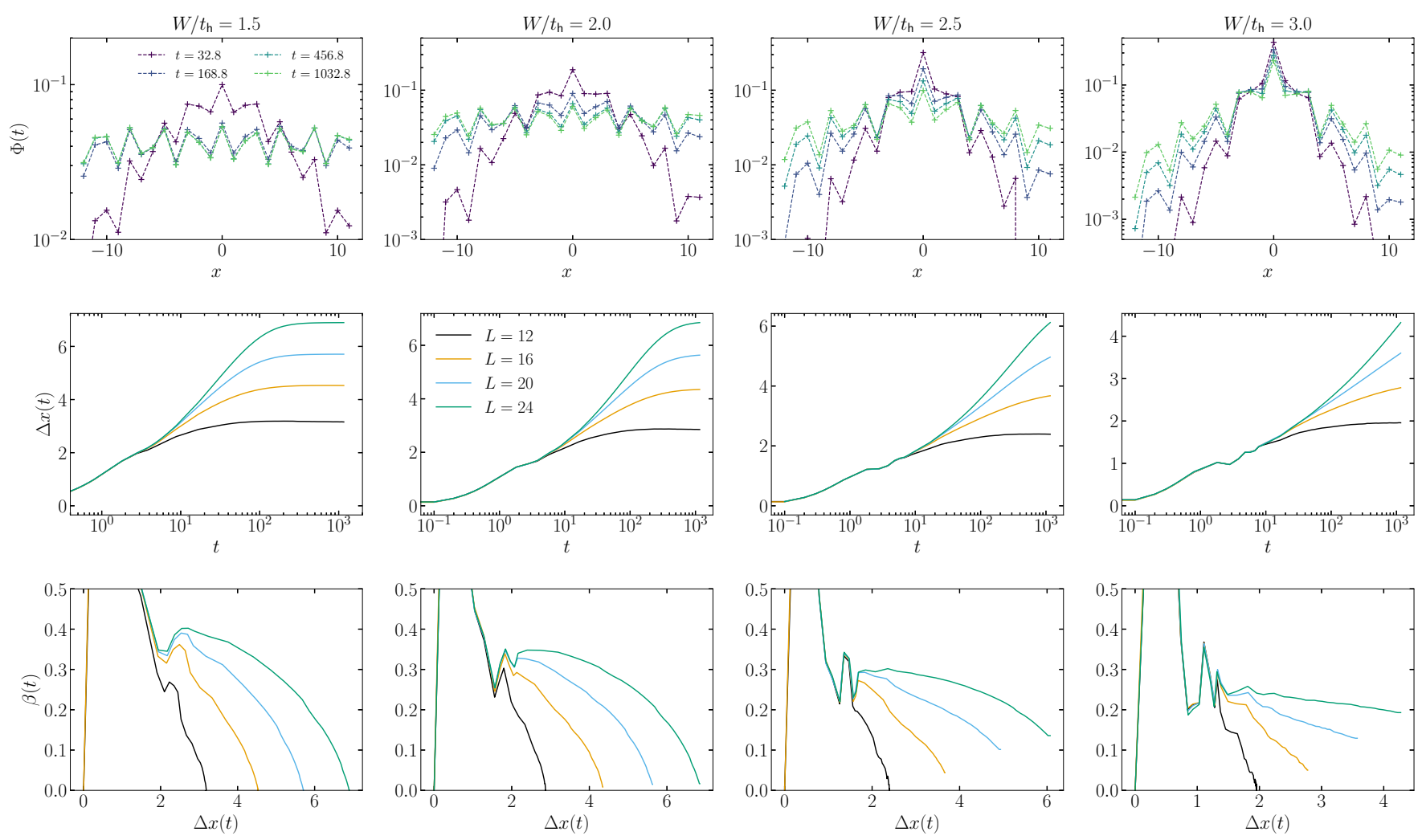

FIG. 10. Top: Shows the evolution of $\Phi(t)$ for different values of disorder $\left(W / t_{\mathrm{h}}=1.5,2.0,2.5,3.0\right)$ at interaction $V / t_{\mathrm{h}}=2.0$ for the AAH model for $L=24$. Inset (b) displays the long-time density correlator that shows pronounce oscillation, which is reminiscent of the underlying quasiperiodic potential (black dashed line). Middle row shows the corresponding $\Delta x(t)$ and the bottom panel displays the evolution of $\beta(t)$ with system sizes $(L=12,16,20,24)$ for different disorder values.

disorder strength could also be dependent on $\alpha: W_{\mathrm{c}}(\alpha)$ [34]. Conceivably, this finding is related to effects such as displayed in Fig. 9.

The analysis of $\Phi(x, t)$ for the quasiperiodic case in terms of the effective exponent $\beta(t)$ is done in analogy to the fully random case. The middle panel of Fig. 10 displays the evolution of $\Delta x(t)$ at system sizes $L=12,16,20,24$ for interaction $V / t_{\mathrm{h}}=2.0$. At small disorder, $\Delta x(t)$ is seen to reach a plateau value $\approx L / 3.5$ indicating saturation due to the finite system size. While the saturation width is only slowly decreasing with disorder, $W$, the saturation time is clearly seen to be a strongly growing function of $W$ exceeding the computational time window already at moderate disorder $W=2.5$.
The bottom panel shows the corresponding evolution of the logarithmic derivative of $\Delta x(t)$, i.e., the exponent function $\beta(t)$. As announced before, the evolution for the quasiperiodic case is analogous to the random disorder case (see Fig. 3, bottom panel). In particular, extremely strong finite-size effects hamper a reliable extraction of the asymptotic exponent. The overall trend is that the short-time exponent at weak disorder takes larger values, which are not far from the diffusive regime, $\beta=1 / 2$.

Strong disorder. At stronger disorder the level statistics is seen to approach the Poissionian limit in (Fig. 8). We thus associate with disorder values $W \gtrsim 5$ a regime close or in the many-body localized phase. Correspondingly, with increasing disorder the dynamics as displayed by $\Delta x(t)$ slows
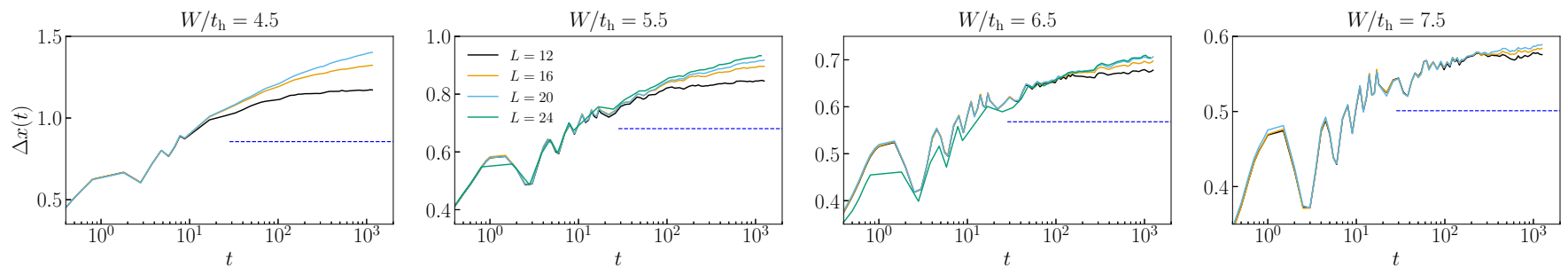

FIG. 11. Shows the time evolution of $\Delta x(t)$ for different values of disorder strength $W / t_{\mathrm{h}}=4.5,5.5,6.5,7.5$ and for interaction strength $V / t_{\mathrm{h}}=2.0$. The dashed line (blue) is indicating the noninteracting value of $\Delta x(t)$ for corresponding disorder strengths for $L=16$ obtained from separate computations. 
down considerably, see Fig. 11; in the quasiperiodic and the fully random situations it shows the same qualitative behavior. Since effects of rare regions are not expected in the quasiperi- odic situation, our observations suggest that the typical strong disorder effects may not be the origin of slow dynamics and creep.
[1] D. M. Basko, I. L. Aleiner, and B. L. Altshuler, Ann. Phys. (NY) 321, 1126 (2006).

[2] I. V. Gornyi, A. D. Mirlin, and D. G. Polyakov, Phys. Rev. Lett. 95, 206603 (2005).

[3] M. Žnidarič, T. Prosen, and P. Prelovšek, Phys. Rev. B 77, 064426 (2008).

[4] A. Pal and D. A. Huse, Phys. Rev. B 82, 174411 (2010).

[5] J. H. Bardarson, F. Pollmann, and J. E. Moore, Phys. Rev. Lett. 109, 017202 (2012).

[6] R. Nandkishore and D. A. Huse, Ann. Rev. Condens. Matter Phys. 6, 15 (2015).

[7] E. Altman and R. Vosk, Ann. Rev. Condens. Matter Phys. 6, 383 (2015).

[8] D. J. Luitz, N. Laflorencie, and F. Alet, Phys. Rev. B 91, 081103(R) (2015).

[9] S. Bera, H. Schomerus, F. Heidrich-Meisner, and J. H. Bardarson, Phys. Rev. Lett. 115, 046603 (2015).

[10] M. Žnidarič, A. Scardicchio, and V. K. Varma, Phys. Rev. Lett. 117, 040601 (2016).

[11] J. H. Bardarson, F. Pollmann, U. Schneider, and S. Sondhi (eds.), Ann. Phys. (Berlin) 529, 1770050 (2017).

[12] F. Alet and N. Laflorencie, C. R. Phys. 19, 498 (2018).

[13] D. A. Abanin, E. Altman, I. Bloch, and M. Serbyn, Rev. Mod. Phys. 91, 021001 (2019).

[14] M. Schreiber, S. S. Hodgman, P. Bordia, H. P. Lüschen, M. H. Fischer, R. Vosk, E. Altman, U. Schneider, and I. Bloch, Science 349, 842 (2015).

[15] J.-Y. Choi, S. Hild, J. Zeiher, P. Schauß, A. Rubio-Abadal, T. Yefsah, V. Khemani, D. A. Huse, I. Bloch, and C. Gross, Science 352, 1547 (2016).

[16] H. P. Lüschen, P. Bordia, S. Scherg, F. Alet, E. Altman, U. Schneider, and I. Bloch, Phys. Rev. Lett. 119, 260401 (2017).

[17] P. Bordia, H. Lüschen, S. Scherg, S. Gopalakrishnan, M. Knap, U. Schneider, and I. Bloch, Phys. Rev. X 7, 041047 (2017).

[18] T. Kohlert, S. Scherg, X. Li, H. P. Lüschen, S. Das Sarma, I. Bloch, and M. Aidelsburger, Phys. Rev. Lett. 122, 170403 (2019).

[19] M. Rispoli, A. Lukin, R. Schittko, S. Kim, M. E. Tai, J. Léonard, and M. Greiner, Nature 573, 385 (2019).

[20] K. X. Wei, C. Ramanathan, and P. Cappellaro, Phys. Rev. Lett. 120, 070501 (2018).

[21] J. Smith, A. Lee, P. Richerme, B. Neyenhuis, P. W. Hess, P. Hauke, M. Heyl, D. A. Huse, and C. Monroe, Nat. Phys. 12, 907 (2016).

[22] G. Kucsko, S. Choi, J. Choi, P. C. Maurer, H. Zhou, R. Landig, H. Sumiya, S. Onoda, J. Isoya, F. Jelezko, E. Demler, N. Y. Yao, and M. D. Lukin, Phys. Rev. Lett. 121, 023601 (2018).

[23] M. Ovadia, D. Kalok, I. Tamir, S. Mitra, B. Sacépé, and D. Shahar, Sci. Rep. 5, 13503 (2015).

[24] S. Bera, G. De Tomasi, F. Weiner, and F. Evers, Phys. Rev. Lett. 118, 196801 (2017).

[25] R. Vosk, D. A. Huse, and E. Altman, Phys. Rev. X 5, 031032 (2015).
[26] A. C. Potter, R. Vasseur, and S. A. Parameswaran, Phys. Rev. X 5, 031033 (2015).

[27] K. Agarwal, E. Altman, E. Demler, S. Gopalakrishnan, D. A. Huse, and M. Knap, Ann. Phys. (Berlin) 529, 1600326 (2017).

[28] D. J. Luitz and Y. B. Lev, Ann. Phys. (Berlin) 529, 1600350 (2017).

[29] P. Prelovšek, M. Mierzejewski, O. Barišić, and J. Herbrych, Ann. Phys. (Berlin) 529, 1600362 (2017).

[30] S. Iyer, V. Oganesyan, G. Refael, and D. A. Huse, Phys. Rev. B 87, 134202 (2013).

[31] M. Lee, T. R. Look, S. P. Lim, and D. N. Sheng, Phys. Rev. B 96, 075146 (2017).

[32] V. Khemani, D. N. Sheng, and D. A. Huse, Phys. Rev. Lett. 119, 075702 (2017).

[33] F. Setiawan, D.-L. Deng, and J. H. Pixley, Phys. Rev. B 96, 104205 (2017).

[34] E. V. H. Doggen and A. D. Mirlin, Phys. Rev. B 100, 104203 (2019).

[35] Y. Bar Lev, G. Cohen, and D. R. Reichman, Phys. Rev. Lett. 114, 100601 (2015).

[36] Y. B. Lev and D. R. Reichman, Europhys. Lett. 113, 46001 (2016).

[37] K. Agarwal, S. Gopalakrishnan, M. Knap, M. Müller, and E. Demler, Phys. Rev. Lett. 114, 160401 (2015).

[38] S. Gopalakrishnan, M. Müller, V. Khemani, M. Knap, E. Demler, and D. A. Huse, Phys. Rev. B 92, 104202 (2015).

[39] I. Khait, S. Gazit, N. Y. Yao, and A. Auerbach, Phys. Rev. B 93, 224205 (2016).

[40] R. Steinigeweg, J. Herbrych, F. Pollmann, and W. Brenig, Phys. Rev. B 94, 180401(R) (2016).

[41] O. S. Barišić, J. Kokalj, I. Balog, and P. Prelovšek, Phys. Rev. B 94, 045126 (2016).

[42] E. V. H. Doggen, F. Schindler, K. S. Tikhonov, A. D. Mirlin, T. Neupert, D. G. Polyakov, and I. V. Gornyi, Phys. Rev. B 98, 174202 (2018).

[43] P. T. Dumitrescu, A. Goremykina, S. A. Parameswaran, M. Serbyn, and R. Vasseur, Phys. Rev. B 99, 094205 (2019).

[44] Y. B. Lev, D. M. Kennes, C. Klöckner, D. R. Reichman, and C. Karrasch, Europhys. Lett. 119, 37003 (2017).

[45] J. T. Chayes, L. Chayes, D. S. Fisher, and T. Spencer, Phys. Rev. Lett. 57, 2999 (1986).

[46] A. Weiße, G. Wellein, A. Alvermann, and H. Fehske, Rev. Mod. Phys. 78, 275 (2006).

[47] V. Khemani, S. P. Lim, D. N. Sheng, and D. A. Huse, Phys. Rev. X 7, 021013 (2017).

[48] J. Gray, S. Bose, and A. Bayat, Phys. Rev. B 97, 201105(R) (2018).

[49] Z. Lenarčič, E. Altman, and A. Rosch, Phys. Rev. Lett. 121, 267603 (2018).

[50] A. Morningstar and D. A. Huse, Phys. Rev. B 99, 224205 (2019) 
[51] A. Goremykina, R. Vasseur, and M. Serbyn, Phys. Rev. Lett. 122, 040601 (2019).

[52] L. Zhang, B. Zhao, T. Devakul, and D. A. Huse, Phys. Rev. B 93, 224201 (2016).

[53] T. Thiery, F. Huveneers, M. Müller, and W. De Roeck, Phys. Rev. Lett. 121, 140601 (2018).

[54] L. Herviou, S. Bera, and J. H. Bardarson, Phys. Rev. B 99, 134205 (2019).

[55] P. Prelovšek and J. Herbrych, Phys. Rev. B 96, 035130 (2017).

[56] M. Mierzejewski, J. Herbrych, and P. Prelovšek, Phys. Rev. B 94, 224207 (2016).

[57] Y. Y. Atas and E. Bogomolny, Phil. Trans. Roy. Soc. A 372, 20120520 (2014).

[58] I. V. Gornyi, A. D. Mirlin, D. G. Polyakov, and A. L. Burin, Ann. Phys. (NY) 529, 1600360 (2017).
[59] N. Macé, F. Alet, and N. Laflorencie, arXiv:1812.10283 [condmat.dis-nn].

[60] M. Serbyn, Z. Papić, and D. A. Abanin, Phys. Rev. X 5, 041047 (2015).

[61] C. L. Bertrand and A. M. García-García, Phys. Rev. B 94, 144201 (2016).

[62] P. Sierant and J. Zakrzewski, Phys. Rev. B 99, 104205 (2019).

[63] K. S. Tikhonov, A. D. Mirlin, and M. A. Skvortsov, Phys. Rev. B 94, 220203(R) (2016).

[64] M. Serbyn, Z. Papić, and D. A. Abanin, Phys. Rev. Lett. 111, 127201 (2013).

[65] D. A. Huse, R. Nandkishore, and V. Oganesyan, Phys. Rev. B 90, 174202 (2014).

[66] D. J. Luitz, N. Laflorencie, and F. Alet, Phys. Rev. B 93, 060201(R) (2016). 